\title{
Il contratto di affiancamento e il subentro nell'azienda agricola
}

\section{Il contratto di affiancamento in agricoltura e il ricambio generazionale}

Per favorire il ricambio generazionale e per sostenere l'occupazione giovanile nel settore agricolo, è stato introdotto dalla legge 27 dicembre 2017, n. 205 il contratto di affiancamento in agricoltura ${ }^{1}$.

La misura s'inserisce nell'ambito dell'evoluzione normativa che ha visto negli ultimi anni, sia a livello comunitario, sia a livello nazionale, numerosi tentativi per promuovere l'occupazione e per rimuovere le barriere all'ingresso dei giovani in agricoltura ${ }^{2}$.

${ }^{1}$ Prima dell'approvazione dell'articolo 1, commi 119 e 120 della Legge 205/2017 (legge di Bilancio 2018), l'articolo 6 della Legge 154/2016 (Collegato agricolo) aveva delegato il Governo del compito di adozione di un decreto legislativo per disciplinare il "contratto di affiancamento in agricoltura", ma tale disposizione non aveva trovato poi immediata attuazione. La differenza di maggiore evidenza è che il nuovo testo prevede l'eventuale stipula del subentro del giovane nell'attività di impresa, o forme di compensazione in caso di conclusione anticipata del contratto, mentre l'articolo 6 della Legge 154/2016, nel dettare i principi della materia, aveva fissato una più ampia gamma di possibilità, una volta concluso l'affiancamento, prevedendo oltre a forme di subentro, anche la trasformazione del contratto di affiancamento in un contratto di conduzione a favore del giovane imprenditore agricolo o in mancanza forme di ulteriori compensazioni a favore del giovane imprenditore agricolo.

${ }^{2}$ I dati relativi all'impegno dei giovani imprenditori in agricoltura vede l'Italia attestarsi al di sotto del valore medio europeo. Cfr. il Documento "Ricambio generazionale in agricoltura e interventi PAC", Rete Rurale Nazionale, Crea e MIPAAF, giugno 2018, p. 5 dove però è specificato che Eurostat definisce giovani coloro che hanno meno di 35 anni, mentre l'Istat considera giovani i soggetti che non hanno ancora compiuto 40 anni. I giovani imprenditori agricoli italiani provengono soprattutto da famiglie agricole che hanno già una disponibilità di terreni e presentano una formazione medio-alta ed una elevata preparazione specialistica. 
Infatti, già in precedenza, con il Regolamento Omnibus e con gli interventi del c.d. "Pacchetto Campolibero" 3 erano stati introdotti strumenti per la semplificazione dell'accesso al credito e agevolazioni alle nuove imprese, attraverso detrazioni fiscali ed un più facile sfruttamento delle risorse fondiarie ed è in questa stessa direzione che si pone la disciplina del contratto di affiancamento in agricoltura.

Il vantaggio derivante dalla stipula di contratti di affiancamento è offerto non soltanto dalla possibilità di collegare questa operazione con la concessione di mutui agevolati a tasso zero per l'impresa affiancata, ma anche dalla possibilità di realizzare notevoli obiettivi di formazione e crescita occupazionale.

Il legislatore italiano ha previsto che i giovani di età compresa tra i diciotto e i quarant'anni, eventualmente organizzati in forma associata, possano stipulare con imprenditori agricoli o coltivatori diretti, di età superiore a sessantacinque anni o pensionati, un contratto di affiancamento comprendente la ripartizione degli utili di impresa a loro favore, in percentuali comprese tra il 30 ed il 50 per cento, purché i soggetti beneficiari non siano titolari del diritto di proprietà o di diritti reali di godimento ${ }^{4}$ su terreni agricoli ${ }^{5}$.

Non si riesce a comprendere bene la ratio di questa rigida scelta di limitazione dei requisiti soggettivi del giovane affiancante, perché potrebbero esistere situazioni in cui sarebbe stato certamente preferibile se il legislatore avesse ammesso i titolari del diritto di proprietà o di diritti reali di godimento sugli stessi terreni agricoli, ponendo magari il divieto soltanto per le ipotesi di proprietari e titolari di diritti reali su fondi diversi rispetto a quello oggetto dell'affiancamento.

Pensiamo, ad esempio, all'ipotesi in cui, da una parte, l'imprenditore da affiancare sia affittuario del fondo, e per ragioni di età non voglia più proseguire l'attività agricola, e dall'altra parte, vi sia un giovane che abbia ereditato il fon-

${ }^{3}$ La legge 116/2014 aveva introdotto il c.d. "Pacchetto Campolibero", dando così attuazione al Piano di interventi per il settore agricolo e il ricambio generazionale in agricoltura, attraverso due tipologie fondamentali di misure: l'abbattimento delle barriere all'ingresso, con detrazioni sull'affitto dei terreni a favore dei giovani e sgravi fiscali per l'assunzione di nuovi lavoratori, nonché attraverso interventi a sostegno dell'avvio di attività, mediante mutui a tassi agevolati per gli imprenditori con meno di quarant'anni.

${ }^{4}$ La ratio di questa previsione risponde alla necessità di consentire «che l'affiancante conquisti una idonea formazione svolgendo un periodo di dedizione all'attività altrui, idonea all'acquisizione delle tecniche necessarie al proficuo svolgimento di quella propria» secondo G. Petteruti, L'affiancamento in agricoltura previsto dalla Legge di Bilancio per il 2018, Studio n. 25 del 22 febbraio del Consiglio Nazionale del Notariato.

${ }^{5}$ La formula prescelta per individuare i requisiti soggettivi dell'affiancante avrebbe dovuto essere espressa in modo differente, quanto meno escludendo dal divieto alcuni diritti reali di godimento, come la superficie (che non può avere per oggetto piantagioni, stante il divieto dell'art. 956 c.c.) o le servitù inerenti al fondo dominante, quando costituite su un immobile diverso dal terreno agricolo. Su questo punto, cfr. Studio n. 25/2018 del Notariato, cit. 
do stesso. In questo caso, stipulando un contratto di affiancamento, il giovane interessato avrebbe il tempo sufficiente per apprendere le tecniche necessarie così da svolgere in futuro l'attività agricola nel proprio fondo.

Del resto, il divieto adottato, che richiama la proprietà, senza ulteriori specificazioni, impedisce la possibilità di stipulare contratti di affiancamento perfino nelle ipotesi in cui affiancante e affiancato siano comproprietari, in caso di successione ereditaria, a meno di non privilegiare una interpretazione sistematica ed estensiva, in luogo di una interpretazione meramente letterale della norma ${ }^{6}$.

Le previsioni introdotte riguarderanno soltanto i contratti stipulati nel periodo 2018-2020. Tale limite temporale, conferisce alle norme valore eccezionale e permette di considerare le agevolazioni previste compatibili con il divieto generale degli aiuti di Stato ${ }^{7}$, oltre ad inserirsi accuratamente nell'ambito del quadro normativo europeo e delle fasi di programmazione della Politica agricola comune.

Con il contratto di affiancamento il soggetto che viene affiancato si obbliga a trasferire al giovane le proprie competenze, mentre quest'ultimo si obbliga a contribuire direttamente alla gestione dell'impresa, anche attraverso servizi manuali e, con il consenso della controparte, si obbliga ad apportare le innovazioni necessarie per il miglioramento dell'azienda agricola.

Il contratto di affiancamento ha una durata libera, ma non può eccedere il limite dei tre anni. In caso di cessione dell'azienda nell'arco temporale di sei mesi dalla conclusione del contratto di affiancamento, al soggetto che affianca l'imprenditore agricolo o coltivatore diretto con età superiore ai sessantacinque anni è riconosciuto il diritto di prelazione. Tale diritto di prelazione ha le caratteristiche proprie della pretesa riconosciuta dall'articolo 8, comma 1 della legge 590 del 1965, e quindi presenta un contenuto che possiamo considerare di portata più ampia rispetto al diritto di prelazione del confinante previsto dall'articolo 7 della Legge 817/1971.

Non mancano però i problemi di interpretazione e di compatibilità delle nuove norme. Per esempio, non è stato sufficientemente chiarito dal legislatore se gli investimenti produttivi e le innovazioni previste durante il contratto di

${ }^{6}$ Cfr. G. Pisciotta, Brevi riflessioni sul «contratto di affiancamento» in agricoltura, "Diritto agroalimentare" 2018,650 , secondo cui sembrerebbe più ragionevole considerare il limite previsto dalla legge come riferito alla sola piena proprietà esclusiva, ammettendo sia le situazioni di comproprietà, sia la titolarità di quote di proprietà riguardanti gli stessi terreni condotti dall'affiancato.

${ }^{7}$ Nonostante il divieto generale di aiuti di Stato, il TFUE prevede la possibilità di deroghe. Per il settore agricolo e forestale, l'articolo 107, paragrafo 2, lettera b) del TFUE prevede la compatibilità degli aiuti destinati a superare i danni arrecati da calamità naturali o da altri eventi eccezionali. Inoltre, a mente dell'articolo 107, paragrafo 3, lettera c), del TFUE, la Commissione può considerare compatibili con il mercato interno gli aiuti di Stato destinati ad agevolare lo sviluppo economico dei settori agricolo, forestale e delle zone rurali, purché gli interventi non alterino le condizioni degli scambi. 
affiancamento e realizzate attraverso il ricorso a mutui agevolati ovvero ad altre forme di finanziamento debbano essere considerate esclusivamente come erogate a favore dell'azienda dell'affiancato ovvero anche a favore dell'affiancante. Il problema sussiste certamente, se consideriamo che il giovane non è titolare dell'azienda e potrà esserlo soltanto nell'ipotesi di un successivo subentro. La qualificazione del giovane affiancante come i.a.p ${ }^{8}$, già nella fase di formazione, spinge a sostenere la tesi secondo cui, fermo restando che gli incentivi sono finalizzati allo sviluppo dell'azienda affiancata, l'operazione di contribuzione del miglioramento, in pendenza di un contratto di affiancamento, è approvata in un quadro certamente più ampio delle situazioni ordinarie, in cui il giovane è chiamato a svolgere un ruolo di diretto interessato.

Da un'attenta lettura delle disposizioni si può evincere che l'attività di affiancamento non può essere equiparata ad una mera attività di tutoraggio e di apprendistato, ma il rapporto instaurato tra affiancante e affiancato assume un valore più ampio e non dovrebbe rappresentare un ostacolo, il fatto che l'affiancante non assuma la contitolarità dell'impresa affiancata, perché quest'ultimo requisito non è sempre fondamentale ai fini della ricostruzione della nozione di impresa collettiva in ambito agrario.

Un esplicito riferimento a tale considerazione si desume, per esempio, dalla disciplina dell'impresa familiare che, proprio in ambito agricolo, assume natura di impresa collettiva?.

\section{Le difficoltà di inquadramento del diritto di subentro}

Il contratto di affiancamento rientra nell'ambito delle misure dirette ad incentivare l'imprenditoria giovanile e il lavoro nel settore agricolo, ma è evidente che la sua funzione non si esaurisce solamente a questi obiettivi.

${ }^{8}$ La qualifica di Imprenditore Agricolo Professionale è attribuita agli imprenditori in possesso di adeguate conoscenze e competenze professionali che dedicano alle attività agricole, almeno il $50 \%$ del proprio tempo di lavoro e che ricavano, da queste attività almeno il 50\% del proprio reddito da lavoro complessivo. Per le attività svolte in zone svantaggiate i requisiti sono ridotti al $25 \%$.

${ }^{9}$ La dottrina e la giurisprudenza maggioritaria riconoscono natura individuale all'impresa familiare, e natura collettiva all'impresa familiare coltivatrice (art. 48 legge 203/1982). All'impresa familiare coltivatrice si applica l'art. 230 bis c.c. nei rapporti interni e per analogia la disciplina della società semplice nei rapporti esterni. Per la disciplina generale dell'impresa familiare, in caso di decesso del titolare, l'impresa cessa di esistere, mentre nell'impresa familiare coltivatrice, in caso di morte del familiare consorziato, si applica l'art. 2284 c.c. Pertanto, sarà necessario liquidare la quota agli eredi oppure sciogliere la società ovvero continuare la società con gli eredi che vi acconsentono. Cfr. I. Canfora, L'impresa familiare in agricoltura e i lavoratori dell'impresa: Spunti di lettura dal diritto italiano ed europeo, "Rivista di diritto agrario" 2014, p. 316 ss.; F. Prosperi, Impresa familiare, in Il codice civile, Commentario, diretto da Schlesinger, Milano, 2006, p. 40. 
Il contratto di affiancamento deve essere presentato insieme al piano aziendale all'Ismea e può prevedere un regime di miglioramenti fondiari ${ }^{10}$, anche in deroga alla disciplina vigente. La possibilità del subentro e le modalità dell'avvicendamento potranno essere espressamente previste nel contratto di affiancamento più in dettaglio, ed in mancanza, potranno essere applicate le regole proprie della cessione dell'azienda ${ }^{11}$.

Il subentro potrebbe anche avvenire mediante concessione in godimento, qualora l'imprenditore affiancato eserciti l'attività su un fondo altrui. In questo caso, potremmo ravvisare una vera e propria antinomia e quindi un problema di compatibilità rispetto al divieto di subconcessione del contratto di affitto, previsto dall'articolo 21 della legge n. 203 del 1982, a meno di non considerare la nuova disposizione come un'espressa deroga al divieto, dimenticando però che per la validità dei contratti in deroga è necessaria l'assistenza e la partecipazione delle Organizzazioni sindacali maggiormente rappresentative a livello nazionale.

Il contratto di affiancamento che ha natura e funzione differente dal contratto di affitto rustico potrebbe quindi in concreto finire per contrapporsi alle cautele e alle riserve previste dalla legge 203/1982, senza che il concedente possa intervenire, e per questa ragione sarebbe stato preferibile un maggior coordinamento normativo da parte del legislatore.

Molti dei problemi descritti sono però in verità attenuati, se si pone attenzione all'effettivo significato da attribuire alla nozione di subentro.

Così, a ben vedere, se consideriamo il diritto di subentro non come una subconcessione in godimento, ma come una forma di subingresso coattivo, nonvi è cessione del contratto (che richiederebbe peraltro il consenso dell'altra parte) e neanche una forma di subcontratto, ma si tratterebbe più esattamente della espressa sostituzione del subentrante nella medesima posizione del subentrato, e tale subingresso, ammesso in via coattiva dalla legge, non genererebbe nessun corto circuito con il divieto di subaffitto ${ }^{12}$ nel settore dei contratti agrari, perché si tratterebbe di una disciplina relativa ad un diverso ambito.

Siamo quindi di fronte ad una forma eventuale di subingresso automatico coattivo $^{13}$ con caratteristiche simili alla disciplina della successione nei contratti

${ }^{10}$ Cfr. E. Capizzano, Miglioramenti agrari, "Novissimo digesto", Appendice, IV, Torino, 1983, 1347.

${ }^{11} \mathrm{~S}$. Bolognini, Cessione dell'azienda agricola e successione nei contratti, "Rivista di diritto agrario" 2016, p. 530 ss.

${ }^{12}$ Cfr. P. Borghi, Lo scioglimento dell'affitto agrario. Risoluzione del contratto e altre fattispecie di cessazione anticipata del rapporto, Padova 2001, p. 230 ss.; A. Germanò, Divieto di subaffitto, "Giurisprudenza agraria italiana" 1982, p. 245; B. Grasso, La disciplina del subaffitto di fondo rustico dal codice alla recente legislazione speciale, "Rassegna Diritto civile" 1983, p. 361.

${ }_{13}$ Per un approfondimento più in generale delle figure normative di successione coattiva nel rapporto contrattuale cfr. M. Paladini, Il subingresso coattivo nel rapporto contrattuale, Pisa 2019, p. 103 ss. 
stipulati per l'esercizio dell'azienda agricola. Tale configurazione potrebbe essere esclusa soltanto nell'ipotesi di patto contrario tra le parti ovvero per la natura personale del contratto.

L'avvicendamento non sarebbe il risultato della cessione del contratto di affitto (inammissibile nell'ambito dei contratti agrari), ma soltanto un'ipotesi (eventuale) di subingresso che troverebbe legittimazione nell'eccezionale disposizione normativa recentemente introdotta.

In questa ipotesi, rimarrebbe però aperto il problema di proteggere il proprietario del terreno, qualora l'affiancante non offra sufficiente garanzia di regolare adempimento delle obbligazioni derivanti dalla prosecuzione del rapporto di durata, potendo questi agire solo in via preventiva, opponendosi eventualmente all'affiancamento del giovane, ma non potendo agire dopo la stipula del contratto di affiancamento, a causa della previsione di subentro introdotta normativamente.

Tuttavia, nel silenzio della legge sul punto, non c'è motivo di escludere che il proprietario del terreno possa comunque continuare ad avvalersi delle obbligazioni contrattuali sulla responsabilità dell'affiancato, anche dopo il subentro dell'affiancante ${ }^{14}$.

Di contro, però, cessato l'usufrutto o l'affitto dell'affiancato, il rapporto contrattuale non potrebbe continuare a sopravvivere a vantaggio solo del giovane subentrato, oltre il termine dell'usufrutto o dell'affitto.

Per questa ragione, dovrebbero essere previste forme di compensazione del giovane imprenditore. In altre parole, il contratto di affiancamento, prevedendo le ipotesi di scioglimento anticipato del rapporto, dovrebbe poter fissare la remunerazione dell'affiancato e dell'affiancante, compresa la liquidazione per i miglioramenti fondiari previsti dal progetto imprenditoriale.

Pur non prescrivendo espressamente la forma $\operatorname{scritta}^{15} \mathrm{e}$ la trascrizione del contratto di affiancamento, per evitare i problemi sopra ricordati, sarebbe oppor-

${ }^{14}$ Si dovrebbe, però, a questo punto, discutere della natura di tale responsabilità, per poterla configurare come solidale o come sussidiaria. Se consideriamo il subentro come un rapporto derivato ex lege, sganciato dal consenso del ceduto, e tale da inibire perfino ogni possibilità di recesso del creditore, sembrerebbe più corretto accettare la tesi di una responsabilità di tipo solidale. Se invece configuriamo il subentro, a seguito di stipula del contratto di affiancamento, come un trasferimento di tutti i rapporti dell'azienda agricola, si dovrebbe parlare di responsabilità sussidiaria e subordinata (ma non solidale). Soltanto, configurando il subentro come una sostituzione inter partes a carattere speciale e coattivamente imposta dalla legge, si potrebbe eventualmente sostenere la tesi di liberazione del subentrato dai precedenti rapporti. Orbene, il legislatore non ha chiarito bene il punto, che rimane collegato al significato da attribuire alla nozione di subentro, suscettibile di interpretazioni assai diverse tra loro, stando a quanto previsto dalla disciplina in materia di contratto di affiancamento agricolo.

${ }^{15}$ In realtà, è possibile desumere la necessità della forma scritta del contratto di affiancamento in agricoltura indirettamente, considerando l'obbligo di allegazione al piano aziendale da presentarsi presso l'ISMEA. 
tuno redigere il contratto per iscritto e provvedere alla relativa trascrizione, così da rendere conoscibile l'ambito di applicazione anche ai terzi.

I punti da chiarire che il legislatore ha lasciato irrisolti sono numerosi e un maggior raccordo tra le varie norme sarebbe stato necessario. Un altro problema rimasto irrisolto, ad esempio, riguarda la possibilità di applicare le agevolazioni i.a.p. e piccola proprietà contadina, anche se il giovane imprenditore non sia iscritto alla gestione previdenziale ed assistenziale INPS.

\section{Concorso tra prelazioni e diritti del giovane subentrante}

In caso di vendita di terreni agricoli durante il periodo di affiancamento ovvero nei successivi sei mesi, è riconosciuto all'affiancante diritto di prelazione a mente dell'articolo 8, comma 1 della Legge 590/1965.

Il richiamo normativo rende applicabile la relativa disciplina che esclude la prelazione nei casi di permuta, vendita forzata, liquidazione coatta, fallimento, espropriazione per pubblica utilità e nelle ipotesi in cui i terreni sono destinati ad utilizzazione edilizia, industriale o turistica in base ai piani regolatori.

Per quanto riguarda la procedura da adottare, il proprietario deve notificare con lettera raccomandata all'interessato la proposta di alienazione trasmettendo il preliminare di compravendita e il giovane imprenditore interessato deve esercitare la prelazione entro il termine di trenta giorni. Se il proprietario non provvede alla notificazione richiesta ovvero se il prezzo indicato è superiore a quello risultante dalla compravendita, il giovane imprenditore può, entro un anno dalla trascrizione del contratto di compravendita, riscattare il fondo dall'acquirente e da ogni altro successivo avente causa. Se il diritto di prelazione è stato esercitato nei termini previsti, il pagamento del corrispettivo deve essere versato entro tre mesi, che decorrono dal trentesimo giorno dell'avvenuta notifica del proprietario, a meno che non venga pattuito diversamente dalle parti.

Se il giovane imprenditore che ha diritto alla prelazione ha presentato domanda per ottenere un mutuo agrario può beneficiare della sospensione del termine, per non più di un anno, fino a che il mutuo non sia stato concesso.

Qualora il versamento del prezzo pattuito sia stato differito, il trasferimento della proprietà del fondo sarà rimesso a condizione sospensiva del pagamento. I coeredi del proprietario venditore, se sono coltivatori diretti, devono essere comunque preferiti al giovane imprenditore.

$\grave{E}$ bene precisare che il diritto di prelazione riconosciuto al giovane imprenditore non ha per oggetto solamente il fondo rustico, ma l'intero complesso aziendale, perché il fondamento della norma è il riconoscimento, ove possibile, della continuità dell'attività agricola e non la mera proprietà del fondo. Per qu- 
esta ragione, il diritto di prelazione riconosciuto al giovane imprenditore assume un diverso rilievo, richiedendo un unico presupposto: l'esistenza di un valido contratto di affiancamento, senza nessun altro requisito richiamato rispetto alle altre forme di prelazione agraria ${ }^{16}$.

Rimane da capire come debba essere risolto il conflitto o il concorso tra prelazionari aventi titoli differenti ${ }^{17}$ e quindi se debba prevalere il giovane agricoltore o se debbano prevalere altri soggetti, qualora questi ultimi siano titolari di diritto di prelazione sul fondo.

Nel caso di concorso tra confinanti, il criterio per individuare la prelazione dominante deve tener conto in primo luogo dell'omogeneità delle colture, insieme alle caratteristiche topografiche dei terreni, della ricomposizione fondiaria, dello sviluppo aziendale e dell'efficienza tecnico-produttiva.

Questi criteri però non possono essere invocati nel caso di concorso con la pretesa del giovane imprenditore affiancante e in tale ipotesi, occorrerà svolgere un diverso ragionamento che tenga conto dell'impatto della disciplina introdotta.

Se si prende in considerazione come principio dirimente, il riconoscimento in capo al giovane imprenditore di un diritto di prelazione che trova giustificazione

${ }^{16}$ La bibliografia in materia di prelazione agraria è molto ampia. A scopo meramente esemplificativo si rinvia a: G. Casarotto, La prelazione agraria, Trattato di diritto agrario, diretto da L. Costato, A Germanò, E. Rook Basile, Torino, 2011; B. Carpino, Prelazione agraria, Novissimo dig. It. App., V, Torni, 1984, 1144; M. Comporti, Profili generali della prelazione agraria, Rivista diritto agrario, 1998, I, p. 149; L. Garbagnati, C. Cantù, La prelazione agraria, Milano, 2007; D. Calabrese, La prelazione agraria. Una ricostruzione attraverso la giurisprudenza della Cassazione, Padova, 2012; L.V. Moscarini, voce Prelazione, Enc. dir., Milano, 1985, XXXIV, p. 983 ss.; M. Tamponi, Prelazione agraria, Dig. Civ., XVI, Torino, 1996. Di regola, in caso di concorso di prelazioni, a parità di oggetto, è la legge che detta l'ordine di preferenza, attribuendo un diverso grado alle varie situazioni. L'ordine di preferenza stabilito dalla legge è di solito accordato in base al maggior valore che s'intende attribuire ad una determinata pretesa, piuttosto che ad un'altra invocabile da un soggetto diverso. Tuttavia, possono esistere situazioni in cui non sia ravvisabile nessuna preferenza ex lege e per l'interprete diventa assai difficile individuare il corretto percorso da seguire. In queste ipotesi, il concorso effettivo tra norme dovrebbe essere risolto applicando il criterio di specialità.

${ }^{17} \mathrm{Da}$ un lato, esistono prelazioni legali "concorrenti” con quella agraria (ad esempio, le ipotesi di: prelazione riconosciuta dall'art. 15, comma 5 della legge n. 394 del 1991 "Legge quadro sulle aree protette", prelazione ex articolo 732 c.c., prelazione a mente dell'art. 230-bis c.c., prelazione urbano-commerciale ex art. 38 della legge n. 392 del 1978), e da un altro versante, il novero delle c.d. "prelazioni agrarie" è aumentato nel tempo, con l'estensione della prelazione agli i.a.p. ex art. 1, comma 3 della legge n. 154 del 2016, con l'introduzione dell'art. 66 del d.1. n. 1 del 2012 a favore di giovani imprenditori agricoli e, da ultimo, con l'approvazione del contratto di affiancamento ex art. 1, comma 119 della legge n. 150 del 2017. Per un esame delle "tensioni" tra le discipline relative alla pluralità di declinazioni dell' istituto della prelazione agraria si veda: A. Jannarelli, Prelazione agraria o prelazioni agrarie: considerazioni introduttive, Rivista diritto agrario, 2018, p. $141 \mathrm{~s}$. 
nell'esigenza di continuità aziendale e di piena attuazione del programma posto in essere con il contratto di affiancamento, è fuori dubbio che, fermo restando quanto previsto dall'ultimo comma dell'articolo 8 della legge 26 maggio 1965 , n. 590 che vede prevalere i coeredi coltivatori diretti del proprietario venditore, nelle altre ipotesi, il carattere eccezionale della disciplina del contratto di affiancamento, tenuto conto della successione delle norme, dovrebbe spingere l'interprete a considerare predominante la posizione del giovane imprenditore che intende subentrare nell'azienda affiancata.

Più esattamente, si potrebbero prospettare differenti ipotesi di conflitto tra discipline ${ }^{18}$, a seconda che l'ultrasessantenne o pensionato risulti proprietario del fondo o mero affittuario. Nella prima ipotesi, in caso di vendita del fondo, l'interprete dovrebbe essere chiamato a sciogliere il problema di un conflitto tra la prelazione dei confinanti e la prelazione del giovane affiancante, mentre nella seconda ipotesi, laddove il venditore del fondo sia un terzo e l'ultrasessantenne o pensionato svolga la sua attività come mero affittuario, il potenziale conflitto riguarderebbe il giovane agricoltore e l'affittuario stesso.

Orbene, nel primo esempio, il diritto dei confinanti soccomberebbe, applicando analogicamente l'esclusione prevista dall'art. 7 della legge n. 817 del 1971 per la presenza sul fondo di coltivatori diretti, mentre nella seconda ipotesi la pretesa del giovane subentrante riceverebbe una corsia preferenziale, proprio in forza della eccezionalità e temporaneità della disciplina del contratto di affiancamento.

In ambedue le ipotesi, il favor legislativo accordato al giovane dovrebbe essere il criterio guida per l'interprete, fermo restando la necessità di un suo effettivo subentro nell'impresa ${ }^{19}$.

${ }^{18}$ Cfr. G. Pisciotta, Conflitto o concorso fra prelazioni, Rivista diritto agrario, 2018, 309, secondo cui si deve parlare di concorso tra prelazioni «in tutti quei casi in cui la fattispecie consente (o quanto meno sembra consentire) l'applicazione di regole preferenziali diverse poste a tutela di interessi/valori differenti e tra di loro confliggenti, determinando la necessità di un intervento interpretativo funzionale alla soluzione del conflitto». Più precisamente, l'A. ritiene opportuno distinguere le situazioni di conflitto vero e proprio dalle situazioni di mero concorso normativo. Sono da qualificare come possibili situazioni di conflitto: le prelazioni di più soggetti, tutti titolari ex art. 8 della legge n. 590/1965; le prelazioni di proprietari coltivatori diretti o i.a.p. confinanti con il fondo in vendita; le prelazioni di soggetti confinanti, mentre sono da qualificare come ipotesi di concorso tra prelazioni agrarie differenti le situazioni che vedono competere tra di loro, i soggetti insediati sul fondo (e dunque titolari del diritto di prelazione agraria) o i proprietari coltivatori diretti o i.a.p. confinanti con i giovani imprenditori agricoli che stipulano contratti di affiancamento.

${ }_{19}$ Cfr. Pisciotta, Conflitto o concorso fra prelazioni, cit., 2018, 324-325, secondo cui, accettando una interpretazione che prediliga la portata eccezionale e temporanea della previsione del comma 119, dell'articolo 1, della legge n. 150 del 2017, la prelazione non potrebbe essere esercitata congiuntamente dagli aventi diritto, in base ai criteri definiti dall'art. 8 della legge n. 590 del 1965. 


\section{L'accesso dei giovani in agricoltura e gli obiettivi dell'Unione europea}

Il ricambio generazionale è un obiettivo fondamentale per la PAC post 2020 anche se nessuna delle misure introdotte a livello comunitario o a livello interno si è dimostrata decisiva. La relazione speciale della Corte dei Conti Europei del giugno $2017^{20}$ ha rilevato numerosi punti deboli che sono risultati dalla scelta del legislatore europeo di aver privilegiato "misure di pagamento" dei giovani, piuttosto che obiettivi di ricambio generazionale o di successione nell'azienda, perseguendo politiche a corto raggio, senza una prospettiva di contenuto più ampio.

Un enorme passo avanti nella programmazione degli obiettivi concreti è stato compiuto con la Comunicazione su 'Il futuro dell' alimentazione e dell'agricoltura" del 29 novembre $2017^{21}$ che ha segnalato il ricambio generazionale come una priorità del futuro quadro politico dell'Unione, da perseguire, non più soltanto attraverso pagamenti mirati al sostegno dell'avviamento aziendale, ma anche attraverso la formazione delle competenze più utili ad assicurare l'ingresso dei giovani nel settore agricolo. Ė evidente però che il raggiungimento di un'efficace politica di ricambio generazionale a livello europeo deve coordinarsi inevitabilmente con le politiche nazionali, perché è proprio da queste ultime che dipendono le regole in tema di previdenza, di lavoro, di accesso al sistema creditizio e fiscale.

Un'altra tappa fondamentale di questo percorso è stata così compiuta con la Risoluzione "Sull'attuazione degli investimenti PAC a favore dei giovani agricoltori nell'Unione europea dopo la riforma 2013", del Parlamento europeo, diretta a limitare lo spopolamento rurale e a incentivare la crescita socio-economica dei territori più svantaggiati.

Le successive proposte di regolamento sulla PAC post 2020 hanno però ridimensionato il contenuto sia della Comunicazione sia della Risoluzione, mantenendo ferma la possibilità per i singoli Stati di promuovere le misure ritenute più idonee per favorire l'accesso dei giovani nel settore agricolo.

Tuttavia, la scelta di adottare prevalentemente programmazioni strategiche nazionali, se da un lato, ha avuto il vantaggio di promuovere misure più vicine alle esigenze dei territori e alla specifica fisionomia delle imprese agricole interne, dall'altro campo ha impedito un più efficace coordinamento normativo.

${ }^{20}$ Cfr. la Relazione speciale della Corte dei Conti: 'Rendere più mirato il sostegno dell’Unione europea ai giovani agricoltori per promuovere efficacemente il ricambio generazionale, giugno 2017.

${ }^{21}$ COM (2017)713final. 
Del resto, la mancanza di un quadro generale di servizi e assistenza tecnica di subentro nella gestione delle imprese è stato il maggior ostacolo al trasferimento delle competenze e alla razionalizzazione degli interventi.

A questa riflessione che riguarda il quadro strategico, va aggiunta una osservazione suppletiva sulle specifiche barriere in Italia. Nel nostro paese, le difficoltà di accesso ai fattori produttivi rimangono per i giovani un problema irrisolto. A questo dato deve aggiungersi la maggiore concorrenzialità di altri settori come il turismo e il commercio che avendo una maggiore redditività rimangono più attraenti per $i$ giovani.

Al contrario, l'obiettivo del rinnovamento generazionale ${ }^{22}$ deve puntare in primo luogo sulla crescita e sulla formazione di nuovi imprenditori, ma anche su un più facile accesso ai fattori produttivi, non soltanto mediante incentivi di tipo episodico, ma attraverso l'aggiornamento e l'attualizzazione delle norme sui contratti agrari ${ }^{23}$, in considerazione di una realtà profondamente mutata rispetto al passato. E si può affermare con certezza che senza una riforma strutturale e completa dell'intera materia, la singola previsione del contratto di affiancamento non potrà essere considerata uno strumento realmente efficace.

\section{THE AID CONTRACT AND THE GENERATIONAL TURNOVER IN AGRICULTURE}

\section{Summary}

The legal framework of the State budget for the year 2018 in Italy provided for measures to encourage the training and development of young entrepreneurs in order to help in the transfer of new generations into agricultural business management. Young people, aged between eighteen and forty, my now enter into an agreement with agricultural entrepreneurs who are over sixty-five years old or retired, to cooperate and share the business profits. During the period of coaching the young person will have to acquire the necessary skills. The contract may provide for eventual takeover by the young agricultural entrepreneur of the management of the business. The author examines the compatibility of the new rules with the agrarian law, showing some weak points that the legislator should have considered.

${ }^{22}$ Tra le misure interne varate per favorire il ricambio generazionale possiamo certamente annoverare la 1.14 febbraio 2006, n. 55 che ha introdotto nel codice civile le norme sul patto di famiglia. Cfr. L. Russo, Patto di famiglia e azienda agricola, "Rivista diritto civile" 2007; G. Petrelli, La nuova disciplina del "patto di famiglia», "Rivista notariato" 2006, 401.

${ }^{23}$ Per un approfondimento: R. Alessi, G. Pisciotta, I contratti agrari, Trattato di diritto civile e commerciale, già diretto da A. Cicu, F. Messineo, L. Mengoni, continuato da P. Schlesinger, Milano 2015, 178 ss. 


\title{
IL CONTRATTO DI AFFIANCAMENTO \\ E IL SUBENTRO NELL'AZIENDA AGRICOLA
}

\author{
Riassunto
}

La legge del Bilancio 2018 ha approvato regole per favorire lo sviluppo di giovani imprenditori e per aiutare il passaggio delle nuove generazioni nella gestione delle imprese agricole. I giovani, di età compresa tra i diciotto e i quaranta anni, possono stipulare con imprenditori agricoli, di età superiore a sessantacinque anni o pensionati, un contratto per dividere gli utili d'impresa. Nel periodo di affiancamento il giovane dovrà acquisire le competenze necessarie e il contratto può stabilire il subentro del giovane imprenditore agricolo nella gestione dell'azienda. L'autore esamina la compatibilità delle nuove norme con le disposizioni della legge agraria, mostrando alcuni punti deboli che il legislatore avrebbe dovuto considerare. 